\title{
Multilinguales
}

\section{Les proverbes et l'air du temps}

Proverbs and Climatic Anomalies

\section{Sonia Fournet-Perot}

\section{(2) OpenEdition}

Journals

Édition électronique

URL : http://journals.openedition.org/multilinguales/2215

DOI : $10.4000 /$ multilinguales.2215

ISSN : 2335-1853

\section{Éditeur}

Université Abderrahmane Mira - Bejaia

\section{Édition imprimée}

Date de publication : 1 décembre 2013

Pagination : 37-42

ISSN : 2335-1535

\section{Référence électronique}

Sonia Fournet-Perot, "Les proverbes et l'air du temps », Multilinguales [En ligne], 2 | 2013, mis en ligne le 01 décembre 2013, consulté le 17 septembre 2019. URL : http://journals.openedition.org/ multilinguales/2215; DOI : 10.4000/multilinguales.2215

Ce document a été généré automatiquement le 17 septembre 2019.

\section{(c) (i) (3)}

Multilinguales est mise à disposition selon les termes de la Licence Creative Commons Attribution Pas d'Utilisation Commerciale - Pas de Modification 4.0 International 


\title{
Les proverbes et l'air du temps
}

\author{
Proverbs and Climatic Anomalies
}

Sonia Fournet-Perot

\section{Les proverbes et l'air du temps}

1 C'est notre expérience du monde qui nourrit la sagesse populaire. A l'origine de tout proverbe, il y a un premier énonciateur qui a cherché à normaliser son quotidien : «la autoría corresponde, porlomenos en su nacimiento, a un individuoconcreto que es quienloemiteporvez primera comoresultado de sus vivenciascotidianas y de su reflexión $»^{6}$ (Ruiz Moreno, 1998: 169). Il est ensuite progressivement effacé sous une kyrielle d'énonciateurs postérieurs pour devenir voix collective et anonyme.

2 Il semblerait que notre environnement (le contexte situationnel) ait ainsi alimenté, au fil des siècles, la matière proverbiale à laquelle nous recourons. Mais que se passe-t-il lorsque celui-ci change et que le langage proverbial ne le légitime plus?

3 C'est à cette question que nous nous efforcerons de répondre à partir d'un cas concret de création proverbiale impulsée par un changement climatique observé en 2013, en Europe : l'hiver s'est prolongé de façon inhabituelle, réduisant le printemps à une peau de chagrin. Ce bouleversement contextuel a donné lieu à une faille cognitive, aucun principe général existant n'étant apte à expliquer la situation vécue. En Espagne, de nouveaux proverbes ont vu le jour pour pallier ce manque.

4 Ces nouvelles séquences, pour provoquer l'engouement, ont dû tenir compte du savoir partagé et respecter le moule propre au langage proverbial; ce qui leur a permis une renommée rapide, facteur de figement progressif et de proverbialisation (Shapira, 2000 : 81-97). L'expérience nouvelle, traduite en proverbes, est alors présentée non plus comme originale mais comme habituelle; corrélativement, d'un point de vue pragmatique, une nouvelle règle d'inférence - un nouveau préconstruit culturel - est venue recréer le lien logique défaillant. 


\section{Les contextes de la genèse proverbiale} année sans été. Le nord de l'Espagne a été particulièrement touché par cette vague de froid, aussi longue qu'inattendue, la presse allant même jusqu'à qualifier le Pays Basque de "pays où le soleil est absent" (García, 2013). Ce phénomène météorologique a constitué un contexte - au sens d'environnement, de situation vécue - surprenant, car exceptionnel.

7 Les recueils espagnols (Correas, 1627 ; Bergua, 1946 ; Junceda, 1999 ; Etxabe, 2012...) se sont toujours efforcés de prévoir les évolutions climatiques, sans aucune précision scientifique mais avec un bon sens éprouvé. Et voilà qu'aucun de ces dichos-predicción (CalzacortaElorza, 1999 : 95) n'avait anticipé un printemps et un début d'été hivernaux : les températures de la fin du mois de juin, voire du début du mois de juillet, étant bien en deçà des normales de saison.

8 La réaction ne s'est guère fait attendre: de nouvelles séquences proverbiales sont apparues et se sont propagées sur les réseaux sociaux ${ }^{7}$, jusqu'à atteindre une renommée suffisante pour être citées dans les médias officiels. Nous avons relevé quatre énoncés faisant référence à cet hiver persistant, soit, par nombre décroissant d'occurrences :

- (1) «HastasanFermín, (con) bufanda y calcetín» (Jusqu'à la Saint Firmin, (avec) écharpe et chaussettes). Les fêtes de la Saint Firmin - "Las Sanfermines " - se déroulent chaque année en Navarre du 6 au 14 juillet. Ce nouveau proverbe préconise donc de ne pas se découvrir d'un fil avant la mi-juillet ;

- (2) «Hasta la Virgen de Begoña quitarse el refajo ¡ni de coña!» (Jusqu’à la Vierge de Begoña, enlever son jupon : pas question !). La période de froid annoncée est ici plus longue puisque la Vierge de Begoña n'est célébrée que le 15 août en Biscaye ;

- (3) «LlegaCeledón y nosotros con edredón" (Celedón arrive et nous avons toujours notre édredon). Celedón est le personnage qui ouvre les fêtes de Vitoria (province basque d'Álava) le 5 août ;

- (4) «Antes sacamos a Marijaia que el bikini pa la playa» (Nous sortons Marijaia avant notre bikini pour la plage); ou sa variante plus rare « Sale Marijaia y nosotros sin pisar la playa» (Marijaia sort et nous n'avons pas encore mis un pied à la plage). L'apparition de Marijaia, symbole de la "Semana Grande » de Bilbao, marque le début des festivités tous les15 août.

Nos recherches sur internet laissent entrevoir que le premier énoncé aurait été attesté pour la première fois sur Twitter le 20 avril 2010. La météo n’a effectivement pas été très clémente au printemps ces quatre dernières années, mais pas au point de déclencher un engouement collectif pour cette création gnomique. Cette forme proverbiale est ensuite apparue sporadiquement jusqu'au mois de mars 2013, date à partir de laquelle elle a connu une diffusion d'autant plus rapide et massive que le beau 
temps et la chaleur se faisaient attendre. Au 3 juillet 2013, on comptait plus de 18000 occurrences en ligne.

Bien que l'origine de ce proverbe remonte à 2010, il aura fallu attendre le premier semestre 2013 et son désordre climatique inédit pour que la propagation de la construction soit suffisante et conduise à son figement et, par voie de conséquence, à sa proverbialisation. La première trace des deux proverbes suivants (2 et 3 ) date respectivement des 15 et 21 mars 2013. Quatre mois plus tard, le deuxième atteignait environ 3500 occurrences et le troisième presque 2000. Son processus de proverbialisation est moins avancé que pour le premier, "HastasanFermín, (con) bufanda y calcetín », mais est cependant enclenché. Ces trois énoncés sont d'ailleurs fréquemment associés :

Las adversas condiciones meteorológicas vienen azotando a la sufrida población desde el pasado año y han ensombrecido un mes de mayo en el que ya debería lucir el sol. Los internautas han decidido consolarse al menos con una sonrisa, y en los últimos días las redes sociales y los programas de mensajería echan humo con imágenes, chistes y montajes. «De los creadores de "Hasta San Fermín con bufanda y calcetín”y ... "Hasta la Virgen de Begoña quitarse el refajo ¡ni de coña!" ... se estrena ... "Llega Celedón y nosotros con edredón" ». Este es un ejemplo más de las ocurrentes chanzas que se intercambian en Whatsapp8. (NoticiasdeNavarra.com, 23/05/2015)

11 La quatrième séquence (4), pourtant plus ancienne (17 février 2013) que les deux précédentes, atteint tout juste 300 occurrences et ne pourra être qualifiée de " proverbe » que si sa forme se fige et que sa notoriété s'accroît. Afin que ces conditions (figement et notoriété) soient remplies, le créateur d'un proverbe potentiel doit faire appel à une imagerie et/ou à des concepts suffisamment généraux pour que le signifié soit intelligible pour le plus grand nombre.

\section{Le contexte cognitif partagé comme baromètre de diffusion}

12 La communication n'est en effet possible entre un locuteur et un interlocuteur que s'ils partagent, non seulement la même langue, mais aussi un ensemble de connaissances auxquelles ils peuvent faire référence. Ces croyances communes au locuteur et à l'interlocuteur constituent ce que Sperber et Wilson (1989) nomment « contexte » dans leur théorie de la pertinence. Ce contexte n'est donc pas une donnée constante, mais est incident aux savoir et expérience de chacun.

Pour qu'un énoncé soit pertinent dans son contexte, c'est-à-dire pour qu'il produise l'effet recherché tout en demandant un minimum d'efforts de traitement, ce dernier doit être commun aux deux acteurs de la communication. Or, les quatre proverbes ayant vu le jour à la suite des évolutions climatiques exceptionnelles que nous avons évoquées plus haut, ils ne renvoient pas tous à des événements dont la notoriété est identique.

14 Par les différents noms de Saints («San Fermín», "la Virgen de Begoña»), ou de personnages fictifs («Celedón », " Marijaia ») convoqués, il est fait référence à des fêtes populaires espagnoles et, plus précisément, basques. Toutefois, la renommée des «Sanfermines" est internationale. Les premières célébrations en l'honneur de Saint Firmin ont eu lieu dès le XII ${ }^{e}$ siècle à Pampelune, capitale de Navarre (Encyclopaedia Universalis, 2013). Originellement religieuses, ces fêtes ont peu à peu été associées aux foires au bétail et aux corridas et ont pris un caractère festif (Sanfermines.net). Ernest 
Hemingway les rendra célèbres bien au-delà de la péninsule dans son roman Fiesta, publié en 1926. Les festivités du 15 août pour la «Virgen de Begoña » à Bilbao, si elles existent depuis le XVI ${ }^{e}$ siècle (Basilicadebegona.com), sont, en revanche, exclusivement religieuses et n'attirent guère hors de la province de Biscaye. Profanes, les personnages de «Celedón » et de "Marijaia », créés respectivement en 1957 (Efe, 2007) et en 1978 (Rucabado, 2013), sont beaucoup plus récents et, également, moins connus au-delà des frontières basques.

Alors que les fêtes de Saint Firmin sont mondialement réputées, la renommée des autres commémorations est moindre, ce qui pourrait justifier la propagation massive de "Hasta la San Fermín, con bufanda y calcetín ", l'extension du contexte de pertinence de cet énoncé étant beaucoup plus importante. En d'autres termes, l'existence des "Sanfermines " étant un savoir partagé par un nombre de sujets parlants beaucoup plus grand, le triomphe du proverbe s'y référant semble aller de soi.

Cependant, la mutualisation ou non-mutualisation de ces connaissances référentielles n'est pas seule en cause : la propagation de ces énoncés tient aussi beaucoup à la nature qu'on a voulu leur donner. Ce ne sont pas de simples principes généraux, mais des proverbes en puissance.

Or, ces objets linguistiques ont un signifiant particulier et normé, aisément reconnaissable et appartenant également à la mémoire collective, au contexte cognitif d'une communauté linguistique. Ce modèle formel proverbial constitue lui-même un autre contexte que tout créateur se doit de respecter.

\section{Le contexte parémiologique comme facteur d'identification et de figement}

Pour devenir proverbes, ces énoncés doivent en avoir l'apparence : autrement dit, leur signifiant doit être en adéquation avec le moule proverbial. Selon Wolfgang Mieder,

[...] los refranes meteorológicos siguen preferentemente un preciso patrón que se puede resumir con la fórmula"Si A, entonces B"; cada enunciado proverbial está formado por un claro antecedente en la primera parte $\mathrm{y}$, en la segunda, una consecuencia9. (Mieder, $1996: 59$ )

19 En d'autres termes, un contexte particulier est évoqué en A, contexte à partir duquel est établie une prévision en B. Et c'est effectivement ce que nous observons dans deux des quatre structures recensées :

- (1) «HastasanFermín, (con) bufanda y calcetín» (Jusqu'à la Saint Firmin, (avec) écharpe et chaussettes) : Si la Saint Firmin (7 juillet) n'est pas passée, il ne faut pas se découvrir.

- (2) « Hasta la Virgen de Begoña quitarse el refajo ¡ni de coña!» (Jusqu’à la Vierge de Begoña, enlever son jupon : pas question !) : Si la fête de la Vierge de Begoña n'est pas passée (le 15 août), il ne faut pas se découvrir.

En revanche les deux autres séquences ne peuvent se plier à ce type de schéma argumentatif sous-jacent :

- (3) «LlegaCeledón y nosotros con edredón » (Celedón arrive et nous avons toujours notre édredon) : Si Celedónestlà est là (le 5 août), il faut rester couvert.

- (4) «Antes sacamos a Marijaia que el bikini pa la playa » (Nous sortons Marijaia avant notre bikini pour la plage) : *si Marijaia est là (le 15 août), il faut rester couvert.

21 Elles seraient porteuses d'une logique antitopique (Fournet, $2005: 75$ ), consistant en un renversement d'une conclusion attendue et pouvant être schématisée par une tournure 
concessive «bien que A, B » : «bien que Celedón soit là, il faut rester couvert », «bien que Marijaia soit là, il faut rester couvert ». Cet écart quant à la norme formelle pourrait expliquer le succès moindre de ces nouveautés proverbiales.

Au-delà de cette structure logique implicite, les proverbes tendent à privilégier certains patrons syntaxiques spécifiques. Après avoir compulsé divers recueils contemporains (Bergua, 1946; Garza Castillo, 1994 ; Junceda, 1999; Etxabe, 2012), il apparaît que la construction [ « Hasta » + date + « no tequites » + vêtement chaud] existe déjà pour des prescriptions de nature météorologique. Nous rencontrons en effet « Hasta el cuarenta de mayo no tequites el sayo " (Jusqu'au quarante mai n'enlève pas ta veste) ou encore " Hasta San Juan, no tequites el gabán » (Jusqu'à la Saint Jean n'enlève pas ton paletot). Une fois encore, les deux énoncés restants ne suivent aucun modèle proverbial existant. Pourquoi alors les considère-t-on comme des proverbes? Parce qu'ils respectent d'autres règles formelles que l'on associe fréquemment à l'univers proverbial :

- la présence de rimes internes, qu'il s'agisse de rimes assonantes, consonantes ou de la répétition des mêmes phonèmes en fin de mot :

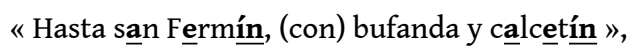

« Hasta la Virgen de Begoña quitarse el refajo ¡ni de coña ! »,

"Llega Celedón y nosotros con edredón ",

" Antes sacamos a Marijaia que el bikini pa la playa ".

L'élaboration de ces énoncés est donc en conformité avec «la fréquente évolution des proverbes vers des structures rimiques » exposée par J.-C. Anscombre (2012: 153). Les rimes sont cependant beaucoup moins recherchées dans la dernière construction, argument supplémentaire à sa propagation plus restreinte.

- La binarité : ces répétitions rimiques contribuent à donner un rythme binaire. Bien qu'il ne s'agisse que d'une vulgate ne concernant pas l'intégralité de la matière proverbiale, cette caractéristique est souvent considérée comme un indice identificatoire. Conformément à cette perception répandue de la «sagesse des nations ", chacune des quatre séquences observées se composent de deux parties bien distinctes, mises en évidence par la rime interne, mais également par la présence fréquente d'une ponctuation ou d'une conjonction de coordination marquant la césure $(/ /)$ :

" HastasanFermín, // (con) bufanda y calcetín »,

« Hasta la Virgen de Begoña // quitarse el refajo ¡ni de coña !»,

« LlegaCeledón // y nosotros con edredón ",

"Antes sacamos a Marijaia // que el bikini pa la playa ».

Si ces quatre créations proverbiales ont bien été impulsées par le contexte situationnel (l'imprévu météorologique), elles ont donc également subi l'influence d'un autre contexte, linguistique, celui de la parémiologie et de ses normes formelles. Toutes conseillent de se méfier de la fraîcheur jusqu'en juillet ou en août, mais seul le signifiant des deux énoncés les plus fréquemment cités (1 et 2) est parfaitement adapté au moule proverbial (logique sous-jacente, syntaxe, rimes).

Comme nous l'avions précédemment supposé, le succès de ces "nouveaux proverbes » semble proportionnel au respect du modèle imposé par le contexte linguistique.

Le contexte, s'il est producteur de sens lorsqu'il est circonstanciel, peut aussi, quand il est linguistique, entraver la diffusion d'un signifié dont le signifiant ne serait pas en adéquation avec un archétype formel préétabli et figé. Si les néo-proverbes se doivent, par ailleurs, de renvoyer à un contexte cognitif commun au plus grand nombre pour 
espérer être colportés et perdurer, leur particularité est de constituer eux-mêmes un contexte logique sous-tendant des raisonnements de nature dialectique.

\section{La création proverbiale ou la justification de processus inférentiels inédits}

\section{Le proverbe : le contexte d'un raisonnement}

La matière proverbiale forme un savoir partagé dont la fonction est d'inciter l'interlocuteur à agir ou à penser d'une façon déterminée. Tout proverbe induit donc une conclusion factuelle, c'est-à-dire un acte directif pour reprendre la terminologie searlienne, et intervient de ce fait nécessairement au sein d'un raisonnement, implicite ou explicite.

Les énoncés proverbiaux, porteurs de préconstruits culturels ${ }^{10}$, représentent les croyances d'un peuple. Ils ne peuvent, en conséquence, être considérés comme véridiques, mais comme vraisemblables et permettent de forger des raisonnements dialectiques, visant à persuader ou à convaincre; ces raisonnements peuvent être argumentatifs ou préventifs.

L'argumentation cherche à " provoquer ou [...] accroittre l'adhésion d'un auditoire aux thèses qu'on présente à son assentiment » (Perelman, 1988 : 16). Elle présuppose donc un contact cognitif entre l'orateur et son auditoire, contact qui en fait un processus essentiellement dialogique: un locuteur tente de faire admettre une conclusion factuelle $\mathrm{C}$ à un interlocuteur par le biais d'un argument A. Si l'acte directif est à réalisation immédiate, il est argumentatif. En revanche, s'il vise une action future, éventuelle, hypothétique, il est préventif (Fournet, 2005 : 206). Seule la connaissance du script suivant lequel se déroule l'énonciation peut permettre de distinguer une conclusion préventive d'une conclusion argumentative.

Dans le cadre d'un raisonnement $\mathrm{A} \rightarrow \mathrm{C}$ légitimé par un proverbe $\mathrm{X}$, l'énoncé proverbial constitue le contexte de croyance - selon la terminologie de Sperber et Wilson (1989) qui permet de distinguer avec pertinence un énoncé représentant un argument d'un autre représentant sa conclusion. La matière proverbiale, parole générale, permet en effet de légitimer une action particulière. Ce passage du général au particulier est autorisé par la mise en place d'un mécanisme enthymémique ${ }^{11}$ interlocutif dont le proverbe est la prémisse majeure ; un enthymème étant un type de syllogisme dont les prémisses sont vraisemblables mais non vraies. Si nous prenons l'exemple de "HastaSan Fermín, con bufanda y calcetín ", nous obtenons :

- prémisse majeure: Si la Saint Firmin (7 juillet) n'est pas passée, il ne faut pas se découvrir(X);

- prémisse mineure : La Saint Firmin n'est pas passée $(\alpha)$;

- conclusion : Il ne faut pas te découvrir( $\beta)$.

3 La mineure $(\alpha)$ et la conclusion ( $\beta$ ) sont l'application du proverbe dans le domaine du particulier, ainsi qu'en atteste la présence de la deuxième personne du singulier (Il ne faut pas te découvrir), laquelle est caractéristique du mécanisme logique déclenché par le proverbe, d'où la qualification d'enthymème interlocutif.

Le schéma argumentatif de cette séquence proverbiale est de type « si... il faut... », sans idée de volonté après la conjonction de subordination (*si l'on veut... il faut...). Le 
passage de $\alpha$ à $\beta$ est dans ce cas indirect et nécessite l'enchaînement cognitif, sur le mode de la concaténation, de quatre préconstruits culturels (PC) initiant chacun des processus enthymémiques. Aucun de ces quatre PC ne coïncide avec le proverbe, celuici étant justement formé de leur succession et encadrant de ce fait l'enchaînement ${ }^{12}$ (voir tableau suivant) :

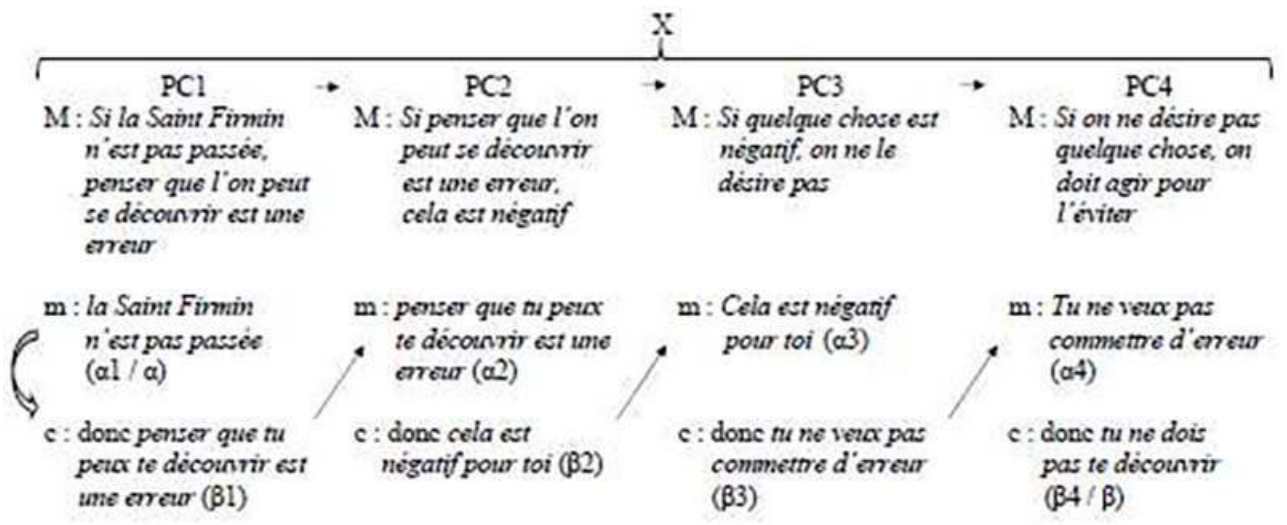

Pour que la conclusion factuelle $\beta 4$ / $\beta$ soit argumentative, elle doit induire l'injonction. Autrement dit, un décodage de l'acte illocutionnaire primaire doit avoir lieu autorisant la transition du DEVOIR FAIRE vers le FAIT. Dans le cas contraire, elle reste préventive.

Si cette concaténation logique, construite à partir du contexte formé par le proverbe, aboutit à la conclusion du raisonnement (C) qu'elle justifie en $\beta 4 / \beta$, elle nous permet également de déterminer ce que serait susceptible d'être l'argument (A) en discours. Il pourrait en effet correspondre à :

- $\alpha 1 / \alpha$ : la Saint Firmin n'est pas passée ( $C=$ alors tu ne dois pas te découvrir);

- $\beta 1$ : penser que tu peux te découvrir est une erreur $(C=$ alors tu ne dois pas te découvrir);

- $\alpha 1 \rightarrow \beta 1$ : la Saint Firmin n'est pas passée ; penser que tu peux te découvrir est donc une erreur ( $\mathrm{C}=$ alors tu ne dois pas te découvrir) ;

- $\beta 3 / \alpha 4$ : tu ne veux pas commettre d'erreur ( $C=$ alors tu ne dois pas te découvrir).

Le proverbe constitue donc un contexte au sein duquel un raisonnement de type $\mathrm{A} \rightarrow \mathrm{C}$ (explicite ou implicite), déterminé par l'intermédiaire d'un processus inférentiel, serait pertinent dans le domaine du particulier. Tout mécanisme argumentatif ou préventif s'appuie ainsi sur un préconstruit culturel dont les proverbes ne sont, en somme, que l'explicitation. La création de séquences proverbiales répondrait, d'un point de vue pragmatique, à une carence logique.

\section{La création d'un nouveau contexte de pertinence}

Comme nous l'avons précédemment souligné, de nouveaux énoncés, calqués avec plus ou moins de succès sur le modèle proverbial, ont vu le jour suite aux très mauvaises conditions météorologiques dont a été victime l'ouest de l'Europe au printemps 2013. Les victimes de ce caprice du climat ont manifesté leur incrédulité, voire leur colère, face à cette situation inhabituelle (De La Fuente, 2013).

Si nous nous intéressons à l'existant, nous observons que les regains de froid au-delà des limites saisonnières de l'hiver ne sont pas exceptionnels, comme en témoignent ces énoncés issus de recueils contemporains : 
- « Hasta el cuarenta de mayo no tequites el sayo » (Jusqu'au quarante mai, n'enlève pas

ta veste),

- «Hasta San Juan, no tequites el gabán» (Jusqu'à la Saint Jean, n'enlève pas ton

paletot).

Le premier énoncé se réfère au 10 juin, le second au 24 juin. D'un point de vue diachronique, on observe une tendance de la froidure hivernale à empiéter de plus en plus sur le territoire de la douceur printanière. En effet, au XVII ${ }^{\mathrm{e}}$ siècle (Correas, 1627), on rencontrait « Hasta mayo no tequites el sayo » (jusqu'au mois de mai n'enlève pas ta veste). Le proverbe actuel, attesté dans l'ensemble des recueils concomitants consultés, prévoit que les températures hivernales peuvent perdurer non plus jusqu'en mai, mais jusqu'à début juin. "Hasta San Juan no tequites el gabán», qui préconise d'attendre fin juin pour enlever son paletot, n'apparaît que chez Etxabe (2012), soit uniquement dans la compilation la plus récente, mettant ainsi en évidence le progressif refroidissement climatique.

41 Les néo-proverbes sont plus pessimistes encore puisqu'ils repoussent l'arrivée définitive de la douceur, au mieux à la mi-juillet, au pire à la mi-août, conformément aux prévisions de Météo France qui annonçait, fin mai, une année 2013 sans été pour l'Europe occidentale. Leur création permet d'instaurer un principe général sur lequel appuyer nos prévisions et nos raisonnements et qui faisait défaut.

L'invention proverbiale permet donc de combler un vide cognitif logique : elle rétablit un contexte dans lequel le raisonnement inédit devient pertinent. La création d'un proverbe est ainsi, avant tout, la création d'un contexte de pertinence visant à justifier une expérience ne cadrant pas avec notre vision du monde. Elle devrait, en conséquence, avoir un effet apaisant puisque qu'elle remet, en quelque sorte, de l'ordre dans notre chaos environnemental et cognitif. Pourtant, ces innovations ont provoqué l'hilarité des interlocuteurs.

\section{L'humour comme échappatoire}

En voici quelques exemples significatifs :

- lorsqu'un auditeur fait mention de " Hasta San fermín, con bufanda y calcetín », dans l'émission de radio El contestador, on entend distinctement les rires des animatrices (Ondacero, 30/05/2005: $01: 05)$;

- il en est de même de l'échange suivant sur hugoymaria.mforos.com :

HOLAAA TXELY..BUENAS NOCHES...conserva los sudores ..que con el frio que hace

falta nos hace...como dicen ahora

Hasta San Fermin con bufanda y calcetin...

Hasta la Virgen de Begoña quitarse el refajo ni de coña...

Llega Celedon y nosotros con edredon...jajaja ${ }^{13}$.

Nous observons le même phénomène sur un blog ${ }^{14}$ :

Ayer leí no sé donde que antes era: "Hasta el 40 de mayo no te quites el sayo", y que ahora es: "Hasta san Fermín con bufanda y calcetín"jajajaja perdonad la gracia mala pero es que es tal cual !15

Les exemples de ce type foisonnent sur les réseaux sociaux. Si ces nouveaux proverbes instaurent un lien logique défaillant, pourquoi provoquent-ils le rire? Il semblerait 
qu'ils établissent un double décalage que nous pourrions qualifier d'hyperbolique. Sont en effet observables :

- une généralisation excessive : en recourant au patron proverbial, on fait d'un événement ponctuel (l'hiver qui se prolonge anormalement) une généralité : le proverbe, stéréotype linguistique, est censé faire état d'un stéréotype culturel et non d'une originalité (climatique) ;

- un ajournement excessif : ces créations renvoient à l'arrivée de la chaleur au mieux à la mijuillet, au pire à la mi-août: il ne s'agit pas de proverbialiser une expérience vécue, mais d'exposer un scénario catastrophe.

Cette exagération est un cas extrême d'usage interprétatif. Le succès de ces séquences a connu un véritable pic en mai 2013, mois habituellement connu pour sa douceur. Pourtant, leur tout premier énonciateur n'a pas choisi de présenter ce même mois de mai, ni même le mois de juin, comme période butoir pour l'avènement définitif des beaux jours. Il a repoussé exagérément cette échéance aux mois d'été. Les prévisions de Météo France ont par la suite - et nous pouvons l'affirmer aujourd'hui - de façon erronée, abondé dans son sens. A une formulation littérale, en adéquation avec la réalité, il a préféré une formulation hyperbolique, plus frappante pour l'interlocuteur et traduisant mieux son intention communicative.

Face à l'impossibilité de bâtir un contexte sérieux où l'exagération trouverait sa place, l'interlocuteur est amené à choisir l'interprétation qui lui coûte le moins d'efforts cognitifs tout en engendrant le maximum d'effets contextuels, celle qui lui semble la plus pertinente: le locuteur polyphonique du proverbe, le "ON-locuteur» (Anscombre, $2000: 11$ ) a sciemment recherché ce décalage hyperbolique. Ces excès, ce grossissement du trait sont révélés par la non-applicabilité de l'énoncé - et ce, dès son énonciation primaire - aux événements expérimentés, événements qui en ont pourtant généré l'émergence.

La création proverbiale, analysée dans le cadre de cet article, nous apparaît ainsi comme une parodie, à savoir un " texte, ouvrage qui, à des fins satiriques ou comiques, imite en la tournant en ridicule, une partie ou la totalité d'une œuvre sérieuse connue » (TLFi, 2002). Le« ON-locuteur " et son destinataire ont trouvé là une cible commune, indispensable à la naissance de l'humour (Charaudeau, 2006: 22), mais aussi à l'adhésion de l'énonciataire. Si un proverbe parodique véhicule toujours un acte directif, par nature excessif (il ne faut pas se découvrir jusqu'au milieu du mois de juillet ou du mois d'août), il implique deux effets perlocutoires successifs distincts ${ }^{16}$ :

- la connivence avec l'interlocuteur, laquelle s'appuie sur la compréhension de l'hyperbole et, en conséquence,

- l'action de l'interlocuteur prenant en compte une atténuation de l'enseignement prodigué : «il ne faut pas se découvrir trop tôt ».

Face à l'adversité et l'« incongruité » climatiques, on dédramatise en parodiant, avec plus ou moins de succès, un langage gnomique connu de tous.

En conclusion, un contexte situationnel particulier a conduit à la conception d'énoncés respectant un contexte linguistique déterminé, celui de l'univers proverbial, et dont la diffusion massive est fonction de l'ampleur du contexte cognitif (au sens sperberien du terme) commun aux différents acteurs de la communication. Le contexte peut donc produire du sens tout en en circonscrivant la forme et en en contraignant la propagation. Mais dans le cas des proverbes, cette génération de sens n'est pas 
anodine: elle déjoue une carence logique contrariant l'appréhension du monde qui nous entoure et nous permet d'en re-formaliser le fonctionnement; car la « sagesse des nations " n'a d'autre fin que la justification de raisonnements, argumentatifs ou préventifs, au sein desquels elle constitue un contexte cognitif de référence autorisant le passage du général (la norme) au particulier (la situation d'énonciation).

Créer un proverbe revient donc à créer un contexte au sein duquel le vécu devient pertinent, où l'expérience inédite fait sens. Il s'agit, en somme, de retrouver des repères. Mais lorsque ceux-ci sont déplaisants, comme c'est le cas dans notre corpus (qui serait adepte d'une nouvelle ère glaciaire?), le nouveau proverbe/contexte peut alors être parodique; l'hyperbole assumée devenant une arme massive de dédramatisation, sans toutefois gommer la fonction didactique intrinsèque au langage proverbial. Le "ON-locuteur» abandonne sa passivité face à l'adversité des événements - fussent-ils simplement météorologiques - et trouve le moyen d'affronter cette réalité grâce à l'humour : "[le] rire [étant] un instrument de survie par sa fonction défensive vis-à-vis de toutes les réalités anxiogènes" (Nezri-Dufour, 2000: 5). Et quel meilleur mariage que celui de l'instruction et de la dérision?

Ces néo-proverbes visent toujours à influencer l'action, physique ou psychique, de l'énonciataire ; mais, en faisant le choix de la formulation hyperbolique, ils gagnent sa connivence, prenant pour cible non seulement le contexte situationnel, à savoir ce printemps "à ne pas mettre un chien dehors", mais également le contexte parémiologique et son utopie totalisatrice. Ne dit-on pas Castigatridendo mores ${ }^{17}$ ?

\section{BIBLIOGRAPHIE}

\section{Corpus}

@Ana M SanchezG, Hugoymaria.mforos, 24/05/2013, disponible sur :

http://hugoymaria.mforos.com/2088279/11079431-hugo-y-maria-6/?pag=18.

«El contestador », in Onda Cero, 30/05/2013, disponible sur :

http://www.ondacero.es/audios-online/herrera-en-la-onda/frikiagenda/contestador-sanfermin-bufanda-calcetin_2013053000081.html.

GARCIA Aitzol, « Euskadi : el país del sol ausente », in Deia, 30/05/2013, disponible sur : http://www.deia.com/2013/05/30/ocio-y-cultura/que-mundo/el-pais-del-sol-ausente

Noticiasdenavarra.com, « Los internautas ya están 'hasta el gorro' del mal tiempo en Navarra », , 23/05/2013, disponible sur :

http://www.noticiasdenavarra.com/2013/05/23/sociedad/navarra/las-redes-sociales-hasta-elgorro-del-mal-tiempo-en-navarra.

«Primavera », in Blog iamabeautyadicta, 05/2013, disponible sur :

http://stylelovely.com/ iamabeautyadicta/2013/05/primavera 


\section{Ouvrages théoriques}

ANSCOMBRE, Jean-Claude, « Parole proverbiale et structures métriques », in Langage, $\mathrm{n}^{\circ}$ 139, Paris, Larousse, Septembre 2000, pp. 6-26.

ANSCOMBRE, Jean-Claude, « Matrices rythmiques et parémies ", in ANSCOMBRE, Jean-Claude, DARBORD Bernard \&ODDo Alexandra (eds), La parole exemplaire, Paris, Armand Colin, 2012, pp. 147-158.

BASILICADEBEGONA.COM, disponible sur :

http://www.basilicadebegona.com/index.html

BERGUA, Juan, Refranero español, 1946, Madrid, Ediciones Ibéricas, Tesoro Literario, 1992.

BERRENDONNER, Alain, Eléments de pragmatique linguistique, Paris, Les Editions de Minuit, Collection Propositions, 1981.

CALZACORTA ELORZA, Javier, « Estructura de los refranes meteorológicos castellanos, franceses y vascos », in Paremia, n 8, 1999, pp. 95-102.

CHARAUDEAU, Patrick, « Des catégories pour l'humour ", in Revue Questions de communication, $\mathrm{n}^{\circ}$ 10, Nancy, Presses Universitaires de Nancy, 2006, pp. 19-41.

CORPAS PASTOR, Gloria, « La creatividad fraseológica: efectos semántico-pragmáticos y estrategias de traducción », in Paremia, n 10, Madrid, 2001, pp. 67-76.

CORREAS, Gonzalo, Vocabulario de refranes y frases proverbiales, 1627, Madrid, Edición Louis Combet revisada por Robert Jammes y Maïté Mir-Andreu, Editorial Castalia, Nueva Biblioteca de Erudición y Crítica, 2000.

DE LA FUENTE, Ilargi Izar, « El mes de agosto no escaparáal mal tiempo », in El País, País Vasco, 20/06/2013, disponible sur :

http://ccaa.elpais.com/ccaa/2013/06/20/paisvasco/1371749592_352681.html

EFE, « Celedón, que cumple 50 años, dará mañana el inicio a las fiestas de Vitoria », in Elcorreo.com, 03/08/2007, disponible sur :

http://www.elcorreo.com/alava/20070803/local/celedon-cumple-anos-dara-200708031247.html ENCYCLOPAEDIAUNIVERSALIS, 2013, disponible sur : http://www.universalis.fr/

ETXABE Regino, Diccionario de refranes comentado, Madrid, Ediciones de la Torre, 2012.

FERNÁNDEZ OCAMPO, AnXo, « (Re)creación involuntaria, atribución e transmisión de paremias no ámbito rural », in Paremia, n 6, 1997, pp. 201-204.

FOURNET, Sonia, Etude descriptive des proverbes dans la littérature hispanique médiévale et pré-classique et de leur fonctionnement au sein des mécanismes de l'argumentation, Thèse sous la direction de Dolorès Ligatto, Limoges, 2005.

GARGALLO GIL, José Enrique, « Dialectología y paremiología : refranes meteorológicos y variación diatópica en la Romania », in Dialectologia, n 7, Universitat de Barcelona, 2011, pp. 37-74.

GARZA CASTILLO, Jorge, Refranero español, 1994, Barcelona, Edicomunicación, Colección Cultura, 1999.

GRIZE, Jean-Blaise, Logique naturelle et communications, Paris, Puf, Psychologie sociale, 1996. JUNCEDA, Luis, Diccionario de refranes, 1999, Barcelona, Espasa Libros, 2012.

MIEDER, Wolfgang, « Los refranes meteorológicos », in Paremia, n 5, Paris, 1995, pp. 59-65. 
NEZRI-DUfOUR, Sophie, «Primo-Levi : rire pour ne pas pleurer », in Italies [En ligne], $\mathrm{n}^{\circ} 4$,

Université de Provence, 2000, disponible sur :

https://journals.openedition.org/italies/2221

PERELMAN, Chaim, L'empire rhétorique, Paris, Librairie philosophique J. Vrin, Collection Pour demain, 1988.

RUCABADO, Beatriz, « Marijaia, un viaje de ida y vuelta », in Elmundo.es, 20/08/2013, disponible sur : http://www.elmundo.es/elmundo/2013/08/20/paisvasco/1376997658.html

RUIZ MORENO, Rosa María, « Reflexiones sobre el origen de los refranes ", inAnaquel de Estudios Arabes, IX, Madrid, 1998, pp. 169-178.

SANFERMINES.NET, Historia de los Sanfermines, disponible sur :

http://www.sanfermines.net/index.php

SCHAPIRA, Charlotte, «Proverbe, proverbialisation et déproverbialisation », in Langage, Paris, $\mathrm{n}^{\circ} 39$, Larousse, 2000, pp. 81-97.

SEARLE John R., Sens et expression, 1979, Paris, Minuit, Le sens commun, 1982.

SPERBER, Dan, WILSONDeirdre, La Pertinence. Communication et cognition, Paris, Minuit, 1989.

TLFi (Trésor de la Langue Française informatisé), 2002, disponible sur :

http://atilf.atilf.fr/tlfv3.htm

\section{NOTES}

6. «L'auteur correspond, du moins au moment de [la] naissance [du proverbe], à un individu concret qui est celui qui le prononce pour la première fois comme résultat de son vécu quotidien et de sa réflexion » (traduit par nous).

7. C'est d'ailleurs par ce biais qu'elles ont été portées à notre connaissance.

8. «Les conditions météorologiques difficiles frappent depuis l'année dernière une population résignée et ont assombri un mois de mai qui devrait déjà voir briller le soleil. Les internautes ont décidé de se consoler au moins avec un sourire et ces derniers jours les réseaux sociaux et les programmes de messagerie se défoulent avec des images, blagues et montages. Des créateurs de “ Hasta San Fermín con bufanda y calcetín" et... "Hasta la Virgen de Begoña quitarse el refajo ¡ni de coña !"... on vous présente ... "LlegaCeledón y nosotros con edredón" ». Voici un exemple de plus des plaisanteries spirituelles qu'on échange sur Whatsapp. » (Traduit par nous).

9. «[...] les proverbes météorologiques suivent essentiellement un modèle précis qui peut être résumé par la formule "Si A, alors B"; chaque énoncé proverbial est composé d'un antécédent évident dans la première partie et d'une conséquence dans la seconde " (Traduit par nous).

10. Il s'agit de " tout un ensemble d'us et de coutumes qui sont inscrits dans la culture à laquelle on appartient » (Grize, 1996 : 66).

11. Le syllogisme, élaboré par Aristote, contraint l'interlocuteur à accepter la thèse proposée en le conduisant de manière nécessaire des principes aux conséquences; il comprend trois propositions : deux prémisses - la majeure et la mineure - et une conclusion. La conclusion est déduite de la majeure par l'intermédiaire de la mineure, ce que l'on affirme légitimement pour un tout pouvant également être affirmé pour les parties de ce tout.

12. En fonction du schéma argumentatif véhiculé - [+/- $\mathrm{P},+/-\mathrm{Q}$ ], [Si/Quand $\mathrm{P}$, alors $\mathrm{Q}$ ], [Bien que $\mathrm{P}, \mathrm{Q}]$ et $[\mathrm{P}>\mathrm{Q}]-$, et de l'expression d'une demande d'action ou d'une idée de volonté au sein de l'énoncé, le proverbe pourra débuter, clore mais aussi encadrer la concaténation enthymémique (Fournet, 2005 : 219-237), comme c'est le cas ici. 
13. «SALUUUT TXELY...BONSOIR...garde tes suées ...parce qu'avec le froid qu'il fait, ça nous manque...comme on dit maintenant: "Jusqu'à la Saint Firmin, avec écharpe et chaussettes... Jusqu'à la Vierge de Begoña, enlever son jupon : pas question...Celedón arrive et nous avons toujours notre édredon...hahaha » (Traduit par nous), @AnaMSanchezG, 24/05/2013 : 23 :07.

14. iamabeautyadicta, $05 / 2013$.

15. "Hier j'ai lu je ne sais où qu'avant c'était: “Jusqu'au quarante mai, n'enlève pas ta veste” mais que maintenant c'est : "Jusqu'à la Saint Firmin, avec écharpe et chaussettes" hahahaha désolée pour la mauvaise blague mais je vous le transmets tel quel ! » (Traduit par nous).

16. Il s'agit de l'effet engendré par la production de l'énoncé sur l'interlocuteur/lecteur.

17. « Il corrige en riant » (Traduit pat nous).

\section{RÉSUMÉS}

Notre vécu et notre environnement spatio-temporel (le contexte situationnel) nourrissent la sagesse populaire. Une situation inédite - le printemps automnal dont a souffert l'Europe de l'Ouest en 2013 - a conduit, en Espagne, à la création de nouveaux proverbes, qui, pour être identifiés et survivre en tant que tels, ont dû respecter un patron formel (le contexte parémiologique) et appartenir au savoir partagé (le contexte cognitif) de la communauté linguistique dont ils sont issus. Ces néo-proverbes ont permis de restaurer une logique mise à mal par une météorologie insolite en établissant, non sans humour, de nouveaux mécanismes inférentiels formant un nouveau contexte cognitif.

Our experience and our spatio-temporal environment (the situational context) nourish popular wisdom. A new situation- the autumnal spring, from which Western Europe suffered in 2013 - led to the creation of new proverbs in Spain. In order to be identified and survive as such, they had to respect a formal model (the paremiologic context) and to belong to the shared knowledge (the cognitive context) of the linguistic community from which they arise. These neo-proverbs allowed the restoration of a logic damaged by an unusual meteorology by establishing, not without humor, new inferential mechanisms which form a new cognitive context.

$$
\begin{aligned}
& \text { إن الواقع المعيش والمحيط الزمكاني (سياق الحال) يغذيان الحكمة الشعبية. فالمثير للدهشة هي تلك الكيال } \\
& \text { الحالة العصيبة التي عاشتها أوروبا الغربية في ربيع } 2013 \text { والذي تمخض الزي المبان بميلاد أمثال شعبية جديدة } \\
& \text { بإسبانيا. }
\end{aligned}
$$

\section{INDEX}

Mots-clés : proverbe, parémiologie, pragmatique, inférence, humour

$$
\text { المثل, مبحث الأمثال, التداولية, الاستدلال, الفكاهةفهرس الكلمات المفتاحية: }
$$

Keywords : proverb, parémiologie, pragmatics, inference, humour 
AUTEUR

SONIA FOURNET-PEROT

Université de Limoges - France 